\title{
Stretching After Heat But Not After Cold Decreases Contractures After Spinal Cord Injury in Rats
}

\author{
Hiroyuki Iwasawa MSc, Masato Nomura MSc, Naoyoshi Sakitani MSc, \\ Kosuke Watanabe MSc, Daichi Watanabe MSc, Hideki Moriyama PhD
}

Received: 10 February 2016/ Accepted: 8 August 2016/Published online: 16 August 2016

(C) The Association of Bone and Joint Surgeons (B) 2016

\begin{abstract}
Background Contractures are a prevalent and potentially severe complication in patients with neurologic disorders. Although heat, cold, and stretching are commonly used for treatment of contractures and/or spasticity (the cause of many contractures), the sequential effects of these modalities remain unclear.

Questions/purposes Using an established rat model with spinal cord injury with knee flexion contracture, we sought to determine what combination of heat or cold before stretching is the most effective for treatment of contractures derived from spastic paralyses and investigated which treatment leads to the best (1) improvement in the loss of
\end{abstract}

One of the authors (HM) has received funding from the Japan Society for the Promotion of Science (JSPS) KAKENHI Grant No. 21700545 (Tokyo, Japan) and The Nakatomi Foundation (Saga, Japan). All ICMJE Conflict of Interest Forms for authors and Clinical Orthopaedics and Related Research ${ }^{\mathbb{R}}$ editors and board members are on file with the publication and can be viewed on request.

Clinical Orthopaedics and Related Research ${ }^{\mathbb{R}}$ neither advocates nor endorses the use of any treatment, drug, or device. Readers are encouraged to always seek additional information, including FDAapproval status, of any drug or device prior to clinical use. Each author certifies that his institution approved the animal protocol for this investigation and that all investigations were conducted in conformity with ethical principles of research.

This work was performed at Kobe University, Kobe, Japan.

H. Iwasawa, M. Nomura, N. Sakitani, K. Watanabe,

D. Watanabe, H. Moriyama $(\bowtie)$

Department of Rehabilitation Science, Graduate School of Health Sciences, Kobe University, Tomogaoka 7-10-2, Suma-ku,

Kobe 654-0142, Japan

e-mail: morihide@harbor.kobe-u.ac.jp

H. Iwasawa

Department of Rehabilitation, St Marianna University School of Medicine, Sugao, Miyamae-ku, Kawasaki, Japan
ROM; (2) restoration of deterioration in the muscular and articular factors responsible for contractures; and (3) amelioration of histopathologic features such as muscular fibrosis in biceps femoris and shortening of the joint capsule.

Methods Forty-two adolescent male Wistar rats were used. After spasticity developed at 2 weeks postinjury, each animal with spinal cord injury underwent the treatment protocol daily for 1 week. Knee extension ROM was measured with a goniometer by two examiners blinded to each other's scores. The muscular and articular factors contributing to contractures were calculated by measuring ROM before and after the myotomies. We quantitatively measured the muscular fibrosis and the synovial intima length, and observed the distribution of collagen of skeletal muscle. The results were confirmed by a blinded observer. Results The ROM of heat alone $\left(34^{\circ} \pm 1^{\circ}\right)$ and cold alone $\left(34^{\circ} \pm 2^{\circ}\right)$ rats were not different with the numbers available from that of rats with spinal cord injury $\left(35^{\circ} \pm\right.$ $\left.2^{\circ}\right)(\mathrm{p}=0.92$ and 0.89 , respectively). Stretching after heat $\left(24^{\circ} \pm 1^{\circ}\right)$ was more effective than stretching alone $\left(27^{\circ} \pm\right.$ $\left.3^{\circ}\right)$ at increasing ROM $(\mathrm{p}<0.001)$. Contrastingly, there was no difference between stretching after cold $\left(25^{\circ} \pm 1^{\circ}\right)$ and stretching alone $(\mathrm{p}=0.352)$. Stretching after heat was the most effective for percentage improvement of muscular (29\%) and articular (50\%) factors of contractures. Although quantification of muscular fibrosis in the rats with spinal cord injury $(11 \% \pm 1 \%)$ was higher than that of controls $(9 \% \pm 0.4 \%)(\mathrm{p}=0.01)$, no difference was found between spinal cord injury and each treatment protocol. The total synovial intima length of rats with spinal cord injury $(5.9 \pm 0.2 \mathrm{~mm})$ became shorter than those of the controls $(7.6 \pm 0.2 \mathrm{~mm})(\mathrm{p}<0.001)$, and those of stretching alone $(6.9 \pm 0.4 \mathrm{~mm})$, stretching after heat $(7.1$ $\pm 0.3 \mathrm{~mm})$, and stretching after cold $(6.7 \pm 0.4 \mathrm{~mm})$ 
increased compared with rats with spinal cord injury ( $\mathrm{p}=$ $0.01, \mathrm{p}=0.001$, and $\mathrm{p}=0.04$, respectively). The staining intensity and pattern of collagen showed no difference among the treatment protocols.

Conclusions This animal study implies that heat or cold alone is ineffective, and that stretching is helpful for the correction of contractures after spinal cord injury. In addition, we provide evidence that heat is more beneficial than cold to increase the effectiveness of stretching.

Clinical Relevance Our findings tend to support the idea that stretching after heat can improve the loss of ROM and histopathologic features of joint tissues. However, further studies are warranted to determine if our findings are clinically applicable.

\section{Introduction}

Joint contractures are a major complication in patients with neurologic disorders [8-10, 15, 20, 46]. Patients with contractures are likely to have pain [10, 26, 37], pressure ulcers [8], and cognitive impairment [26]. Contractures also can contribute to impaired function and activity limitations [5, 12, 14, 16, 19, 26]. Notably, the proportion of patients who have contractures develop in major joints 1 year after spinal cord injury ranges from $11 \%$ to $43 \%$, and it is difficult to accurately predict those susceptible to contracture soon after injury [7]. Therefore, prevention and treatment of contracture are the most important goals in the management of such patients.

A contracture is characterized by limitations in passive ROM, loss of elasticity, and shortening of the soft tissues [3]. Stretching, the process of applying tension to soft tissue structures, is a widely accepted means to treat and prevent contractures. Although much effort has been made to investigate the effectiveness of stretching on contractures or spasticity in human subjects with neurologic disorders, the clinical benefit of these interventions overall is unclear $[4,15,20]$. Some evidence from animal studies suggests that correction of immobilizationinduced contractures can be achieved through passive stretching [6, 11, 36, 38, 42, 44]. However, contractures after central nervous system injury are mediated by not only structural adaptation of soft tissues, but also neural factors including paralysis $[3,25]$. We developed a rat model of contracture after spinal cord injury as a representative animal model of central nervous system injury [28, 30-33], and reported that high-torque and long-duration static stretching led to restoration of the loss of ROM and prevented shortening and adhesions of the joint capsule [29].
In the clinical setting, heat also is used to stretch the affected joint, because it effectively increases the extensibility of the periarticular structures. This concept is based on ex vivo experiments, in which the combination of heat and stretching resulted in elongation of collagenous tissue in the rat tail tendon [23, 45]. Although an in vivo animal study showed that thermal therapy increases the effectiveness of stretching for treatment of contractures after immobilization [43], contractures produced experimentally by immobilizing joints do not model specific contractures in humans with neurologic conditions. In spastic conditions, the consequent motion loss of the muscular imbalance resulting from spasticity is the basis for the contracture development [3]. Heat and cold have a direct effect on the gamma fibers of muscle spindles and reduce its activity and sensitivity to stretch, and as a result, muscle spasm is relieved [24, 39]. In this manner, therapeutic heat and cold before stretching appear to be effective for treatment and prevention of contractures in subjects with spastic paralyses. However, the effectiveness of thermal or cold therapy before stretching is based on clinical experience, and to our knowledge, there is no evidence to support this experience. Whether these modalities are truly worthwhile remains elusive, and the therapeutic effect on the pathophysiology of contractures has received little attention [23, 24, 45].

The purpose of our study was to determine what combination of heat or cold before stretching is the most effective for treatment of contractures after spinal cord injury. Using an established rat model with spinal cord injury and with knee flexion contracture, we investigated which treatment leads to the best (1) improvement in the loss of ROM; (2) restoration of deterioration in the muscular and articular factors responsible for contractures; and (3) amelioration of histopathologic features such as muscular fibrosis and shortening of the joint capsule.

\section{Materials and Methods}

Forty-two 10-week-old male Wistar rats weighting 316 to 404 g (SLC, Japan, Inc, Shizuoka, Japan) were randomly assigned to the following seven equal-sized groups $(n=6$ rats per group): caged control and untreated after spinal cord injury, treated with heat alone, cold alone, stretching alone, stretching after heat, and stretching after cold. The rats in the control group had no intervention, whereas the others underwent spinal cord transection at the level of T8. Surgical procedures and postoperative care confirmed results of previous studies [28-33]. We provided no treatment for 2 weeks postinjury to develop spasticity and contracture [32] and started intervention at postoperative 
Day 15. Rats in the treatment groups received each treatment once daily for 1 week. Given the high cost of maintaining larger species, smaller-animal models are preferred for preliminary screening, and rat models frequently are used for initial assessment of in vivo efficacy. The right and left knees were regarded as different samples ( $\mathrm{n}=12$ limbs per group). The subgroup sample sizes were calculated by a power analysis based on pilot results for detecting a $10^{\circ}$ difference in ROM 19 of 20 times [29, 33].

According to the method described by Usuba et al. [42], stretching was applied while the anesthetized animal was in a supine position with the pelvis stabilized by an elastic tube attached to the treatment table. The ankle was tied to a weight, and then the direction of the stretch was set toward extension with traction applied to the whole lower extremity. The hip was in approximately $100^{\circ}$ to $120^{\circ}$ flexion and was maintained in a neutral position with respect to rotation and abduction and adduction. The stretching condition used in this study was static and the amount of torque was fixed at $0.045 \mathrm{Nm}$; this protocol has been shown to prevent the progression of knee flexion contracture in rats with spinal cord injury [29]. We used a hot bath for thermal therapy and a cold bath for cold therapy. Water temperature for thermal therapy was adjusted to $38^{\circ} \mathrm{C}$, which is higher than the rectal temperature of rats, whereas that for cold therapy was adjusted to $10^{\circ} \mathrm{C}$, both of which are in the nonnoxious range [22]. To preclude the possibility that water-shedding hair of rats reduces the effects of these interventions, the skin was exposed by shaving. The entire hind limbs distal to the hips were immersed in the water. Each treatment was performed for 15 minutes with the rats under anesthesia. Rats in the stretching after heat and cold groups underwent a bath first and then stretching.

At the end of the experimental period, knee motion was measured for extension with the rat under anesthesia according to the previously described method [29, 33]. Briefly, knee motion was measured for extension with a mechanical goniometer applying a standardized torque $(0.06 \mathrm{Nm})$. Two examiners (HM and YT) conducted the ROM measurements and repeated them three times for each leg. The examiners were blinded to each other's scores. Reported values are the mean of the six measurements, the combined measurements taken by both examiners. After the measurements, the animals were euthanized by exsanguination. Myotomy of the knee flexors then was performed, and ROM was measured again in extension. These measurements were conducted three times for each leg, and the mean value obtained was reported as the result. To minimize the effects of rigor mortis on the results, the measurements after the myotomies were completed within 15 minutes after death. According to our previous method [29], the formulas assessed by measuring ROM before and after the myotomies allow isolation of the muscular and articular factors contributing to contractures and are as follows: muscular factors $=$ ROM no myotomy - ROM after myotomy (within each group); articular factors = ROM after myotomy of each group - ROM after myotomy of the control group.

After the ROM measurements, the biceps femoris and whole knees including the capsule were removed and snapfrozen. Cross-sections of $10 \mu \mathrm{m}$ then were prepared from muscle samples. In addition, each sample of the knees was sectioned in $5-\mu \mathrm{m}$-thick slices at the medial midcondylar level in the sagittal plane following the protocols established by Kawamoto [21].

\section{Quantification of Fibrosis in Muscle Tissue}

We evaluated muscular fibrosis, which contributes to poor muscle extensibility in muscular contracture $[2,35]$, by a slight modification of the method according to Hadi et al. [13]. Four samples of biceps femoris were randomly selected from each group. The histologic sections were stained with picrosirius red and digitized by an $\mathrm{x} 10$ microscope objective with a light microscope (BX53; Olympus, Tokyo, Japan) and a camera (DP73; Olympus). Fibrosis was quantified with ImageTool software (Image J $1.47 \mathrm{v}$; National Institutes of Health, Bethesda, MD, USA). Excluding the areas such as staining artifacts or vessels manually, the yellow color of the muscle cells and the red color of connective tissue were identified by threshold color plugin. The area of each color was measured separately. We calculated the percentage of connective tissue area by dividing the red area by the total area. For each section, the measurements were performed at 10 areas randomly selected and the mean value was obtained. Measurements were done by one investigator (HI) and the results were confirmed with a blinded observer (HM).

\section{Synovial Intima Length}

To assess shortening of the joint capsule, which is responsible for articular contracture [29, 31, 40], we measured posterior synovial intima length by the method of Ando et al. [1]. Four samples of knees were randomly selected from each group, and the sections were stained with hematoxylin and eosin. On the histologic pictures photographed through an $\mathrm{x} 4$ microscope objective, the length of the superior and inferior subdivisions of the 
synovial intima in the posterior capsule were measured separately. The synovial lining contour of each subdivision, which is from the synoviocartilage junction of the femur or tibia to the posterior horn of the meniscus, was traced manually with Adobe ${ }^{\circledR}$ Photoshop CS2 (Adobe ${ }^{\circledR}$ Systems Inc, San Jose, CA, USA). The length of the drawn line then was measured with Image J. Measurements were done by one investigator (HI), and the results were confirmed with a blinded observer (HM).

\section{Immunohistochemical Analysis}

We assessed the distribution of the two types of the primary collagen isoforms of skeletal muscle immunohistochemically according to the protocols established in our laboratory [27]. Frozen sections of biceps femoris were incubated with mouse monoclonal antibody against collagen Type I (diluted 1:4,000; C2456; SigmaAldrich, St Louis, MO, USA) or collagen Type III (diluted 1:8,000; C7805; Sigma-Aldrich) followed by biotinylated horse antibody against mouse IgG (diluted 1:250; BA2001; Vector Laboratories, Burlingame, CA, USA). The slides then were reacted with VECTASTAIN ${ }^{\circledR}$ Elite ABC regent (diluted 1:50; PK-6100; Vector Laboratories), and immunoreactivity was observed with 3,30-diaminobenzidine tetrahydrochloride (K3466; Dako Japan, Tokyo, Japan). Counterstaining was performed with hematoxylin. The primary antibody was omitted for the negative controls. Observations were done blindly and in random order by one investigator (HM).

\section{Statistical Analyses}

Results for ROM, percentage of connective tissue area in the muscle tissue, and synovial intima length were analyzed statistically with SPSS Version 21.0 (IBM Japan, Tokyo, Japan). First all data were checked for normality with the Shapiro-Wilk test. When normality was observed in all assays, the results were compared among all groups with ANOVA followed by Tukey's honestly significant difference test. Probability values less than 0.05 were considered significant in these analyses.

Statistical analyses of muscular and articular factors in contractures were performed with Microsoft $^{\circledR}$ Excel $^{\circledR}$ 2010 (Microsoft $^{\circledR}$ Corporation, Redmond, WA, USA). Calculating the SDs for the mean differences among the groups [17], 95\% CIs were estimated. We identified the difference as significant when a length of the $95 \%$ CI did not overlap zero [29, 33, 41].

\section{Results}

Improvements in the Loss of ROM

Knee flexion contractures developed in rats with spinal cord injury (Fig. 1A). When compared with rats untreated after spinal cord injury $\left(35^{\circ} \pm 2^{\circ}\right)$, knee extension ROM improved in rats treated with stretching alone $\left(27^{\circ} \pm 3^{\circ}\right)$, stretching after heat $\left(24^{\circ} \pm 1^{\circ}\right)$, and stretching after cold $\left(25^{\circ} \pm 1^{\circ}\right)($ all $\mathrm{p}<0.001)$, whereas not (with the numbers available) in rats treated with heat alone $\left(34^{\circ} \pm 1^{\circ}\right)$ and
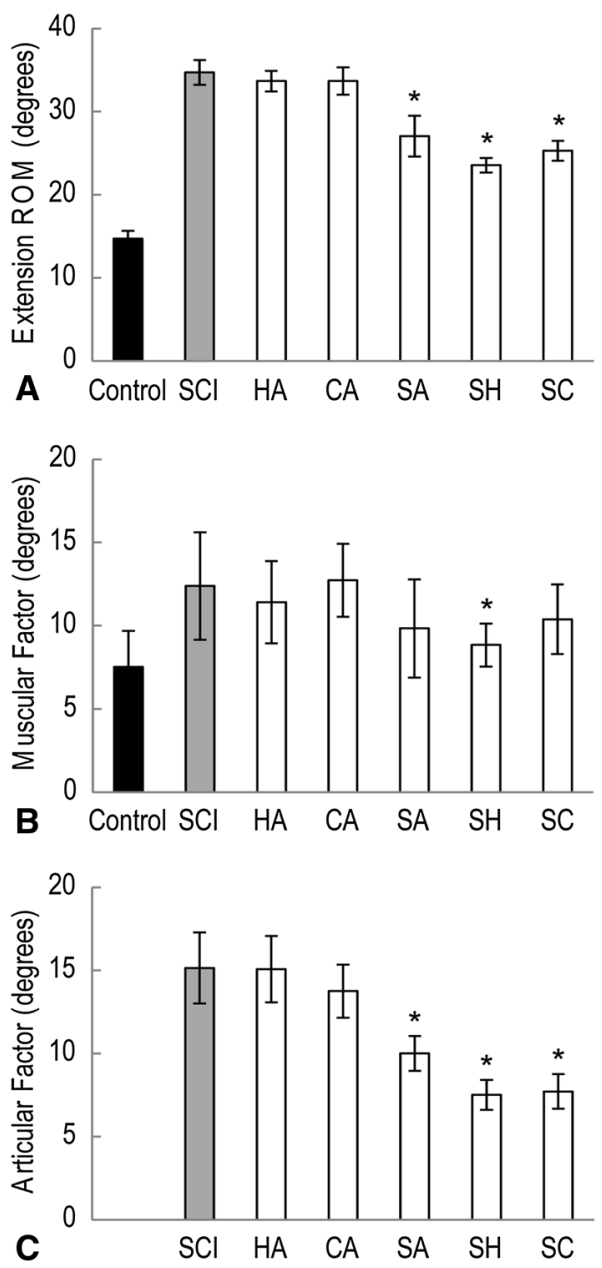

Fig. 1A-C (A) Knee motion in the control group, and spinal cord injury (SCI), heat alone (HA), cold alone (CA), stretching alone (SA), stretching after heat $(\mathrm{SH})$, and stretching after cold (SC) groups was measured for extension with a goniometer. Maximum extension ROM was improved in the stretching alone, stretching after heat, and stretching after cold groups, compared with the spinal cord injury group, but it did not recover to the same extent as in the control group. (B) Muscular factor was improved only in the stretching after heat group, and the other groups were larger than the control group. (C) Articular factor was improved in the stretching alone, stretching after heat, and stretching after cold groups. *Less than the spinal cord injury group. 
cold alone $\left(34^{\circ} \pm 2^{\circ}\right)(\mathrm{p}=0.92$ and 0.89 , respectively). In addition, stretching after heat $\left(24^{\circ} \pm 1^{\circ}\right)$ resulted in better ROM than stretching alone $\left(27^{\circ} \pm 3^{\circ}\right)(\mathrm{p}<0.001)$. However, knee motion of all treated groups did not recover to the same range as that in the control group.

Improvements in the Muscular and Articular Contributions to Contractures

Stretching after heat was the most effective for percentage improvement of muscular (29\%) and articular (50\%) factors of contractures (Table 1). Only stretching after heat $\left(9^{\circ} \pm 1^{\circ}\right)$ accelerated recovery of the muscular factor, when compared with the untreated spinal cord injury group $\left(12^{\circ} \pm 3^{\circ}\right)(95 \% \mathrm{CI}, 0.91-6.18)$ (Fig. 1B). For the articular factors, the stretching alone $\left(10^{\circ} \pm 1^{\circ}\right)$, stretching after heat $\left(8^{\circ} \pm 1^{\circ}\right)$, and stretching after cold $\left(8^{\circ} \pm 1^{\circ}\right)$ groups were smaller than the spinal cord injury group $\left(15^{\circ} \pm 2^{\circ}\right)$ (95\% CI, 3.51-6.76, 5.87-9.38, and 5.61-9.22, respectively) (Fig. 1C). Furthermore, the stretching after heat and cold alone groups had decreased articular factors greater than that of the stretching alone group (95\% CI, 1.50-3.48 and 1.23-3.34, respectively).

\section{Improvements in the Histopathologic Alterations}

Connective tissues in the control biceps femoris were distributed narrowly at the perimysium and endomysium, whereas after spinal cord injury the distribution was denser and wider than in the control group (Fig. 2A-G).

Quantification of the ratio of the connective tissue area to the whole area in an image revealed that the percentage was higher in the spinal cord injury group $(11 \% \pm 1 \%)$ compared with the control group $(9 \% \pm 0.4 \%)(\mathrm{p}=0.01)$. No difference was found between the untreated spinal cord injury group and each treated group (Fig. 2H).

The posterior joint capsule in rats untreated after spinal cord injury exhibited some histologic alterations such as reduction of the synovial fold and joint space narrowing, which resulted from adhesions of the posterosuperior synovial membrane to the posterior femoral cartilage (Fig. 3A-G). These findings also were observed in the heat and cold alone groups, but not in the stretching alone, stretching after heat, and stretching after cold groups.

In line with these observations, the total synovial intima length, which is the sum of the posterosuperior and posteroinferior subdivisions, became shorter after spinal cord injury (Fig. $3 \mathrm{H}$ ), and the length increased in the stretching alone $(6.9 \pm 0.4 \mathrm{~mm})$, stretching after heat $(7.1 \pm 0.3 \mathrm{~mm})$, and stretching after cold $(6.7 \pm 0.4 \mathrm{~mm})$ groups compared with the untreated spinal cord injury group $(5.9 \pm 0.2 \mathrm{~mm})$

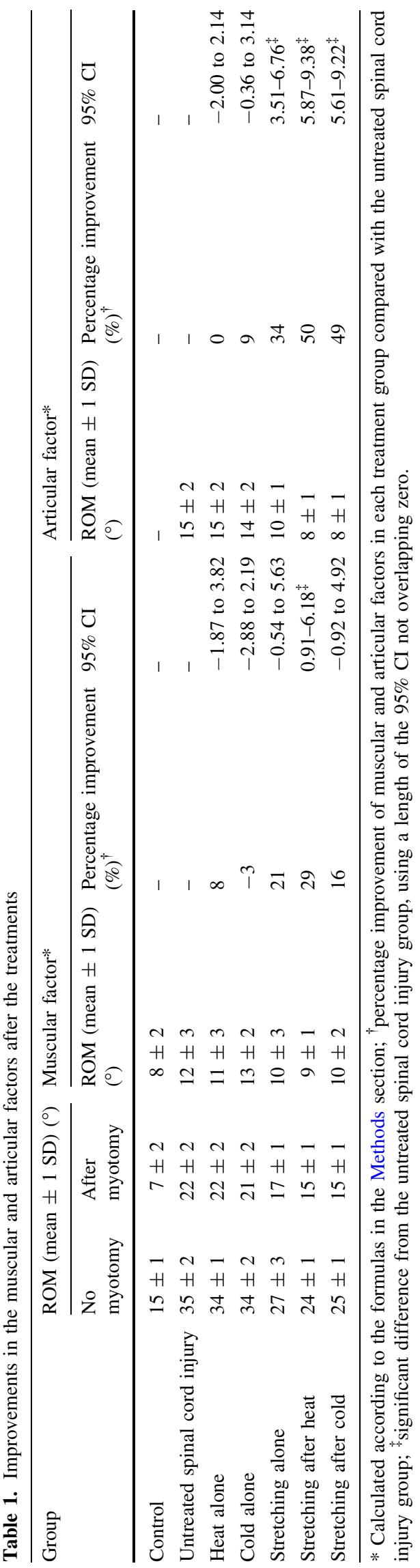



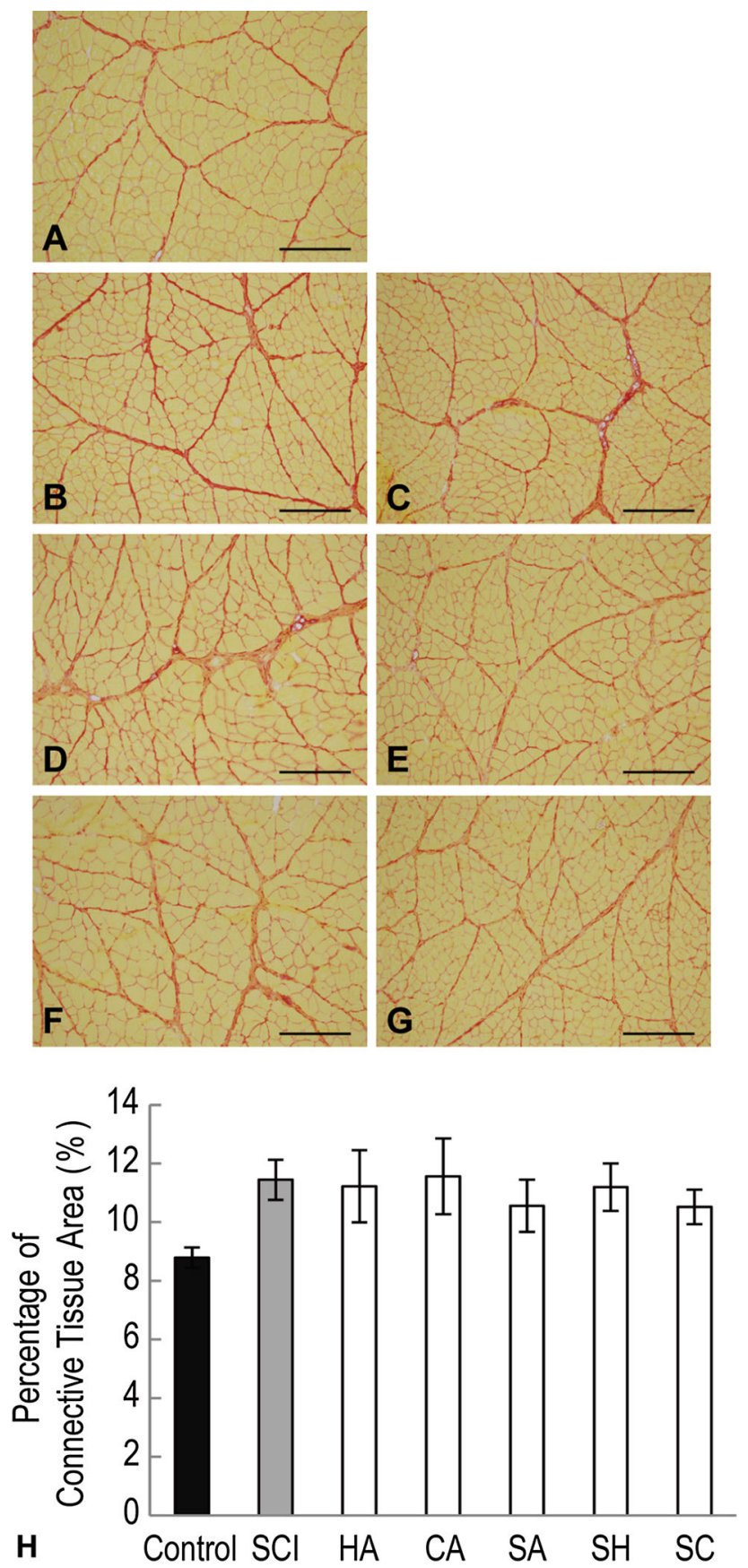

Fig. 2A-H Fibrosis was evaluated on histologic sections stained with picrosirius red in (A) the control group, and the (B) spinal cord injury, (C) heat alone, (D) cold alone, (E) stretching alone, (F) stretching after heat, and (G) stretching after cold groups. Bars $=300$ $\mu \mathrm{m}$. (H) The percentage of connective tissue area in the biceps femoris was calculated. $\mathrm{SCI}=$ spinal cord injury; $\mathrm{HA}=$ heat alone; $\mathrm{CA}=$ cold alone; $\mathrm{SA}=$ stretching alone; $\mathrm{SH}=$ stretching after heat; $\mathrm{SC}=$ stretching after cold.

$(\mathrm{p}=0.01, \mathrm{p}=0.001$, and $\mathrm{p}=0.04$, respectively). Similarly, the posterosuperior synovial intima length improved only in the stretching alone $(5.6 \pm 0.2 \mathrm{~mm})$, stretching after heat $(5.9 \pm 0.2 \mathrm{~mm})$, and stretching after cold $(5.5 \pm 0.5 \mathrm{~mm})$ groups compared with the spinal cord injury group $(4.7 \pm$ $0.2 \mathrm{~mm})(\mathrm{p}=0.01, \mathrm{p}<0.001$, and $\mathrm{p}=0.01$, respectively) (Fig. 3I). For the posteroinferior synovial intima length, however, no differences were found between each treatment group and the untreated spinal cord injury group (Fig. 3J). The total and posterosuperior synovial intima length of the stretching after heat group was longest in all treated groups.

Immunolabeling to collagen Types I and III was detected in all the observed muscle tissues (Fig. 4), except for the negative control. Collagen Type I was distributed predominantly in the perimysium, whereas collagen Type III was seen strongly in the perimysium and endomysium. The staining intensity and pattern showed no difference among the groups.

\section{Discussion}

Heat, cold, and stretching are commonly used for inhibition of spasticity and/or treatment of contractures after a central nervous system injury [24, 39], but whether combinations of these modalities are truly worthwhile remains elusive and the therapeutic effect on the pathophysiology of contractures has received little attention [23, 24, 45]. The goal of our current animal study was to determine what combination of heat or cold before stretching is the most effective for treatment of contractures derived from spastic paralyses. We have shown in a rat model that stretching after heat is the most effective for improvements of knee extension ROM and the muscular and articular factors contributing to contractures. Based on the results of histologic analyses, stretching after heat showed the greatest increases in posterior synovial intima length, and restoration of the ROM is partly explained by the improvements of synovial adhesions and capsular shortening.

Our study has several limitations. Because we used a hot bath as thermal therapy, which was a superficial modality, the thermal stimulation may not affect deeper tissues of muscles or periarticular structures. In addition, although stretching after heat achieved a marked increase in the extension ROM after spinal cord injury, it did not recover to the same extent as that in the normal rat. Given the high cost of maintaining larger species, smaller-animal models are preferred for preliminary screening. The most efficacious compound in a rat model might subsequently be tested in a larger animal model such as rabbits before setting up human trials. Further studies will have to clarify whether similar results are expected in other larger animals. In addition, we used the right and the left knees as different samples. The use of both joints has the advantages of minimizing the number of experimental animals needed 

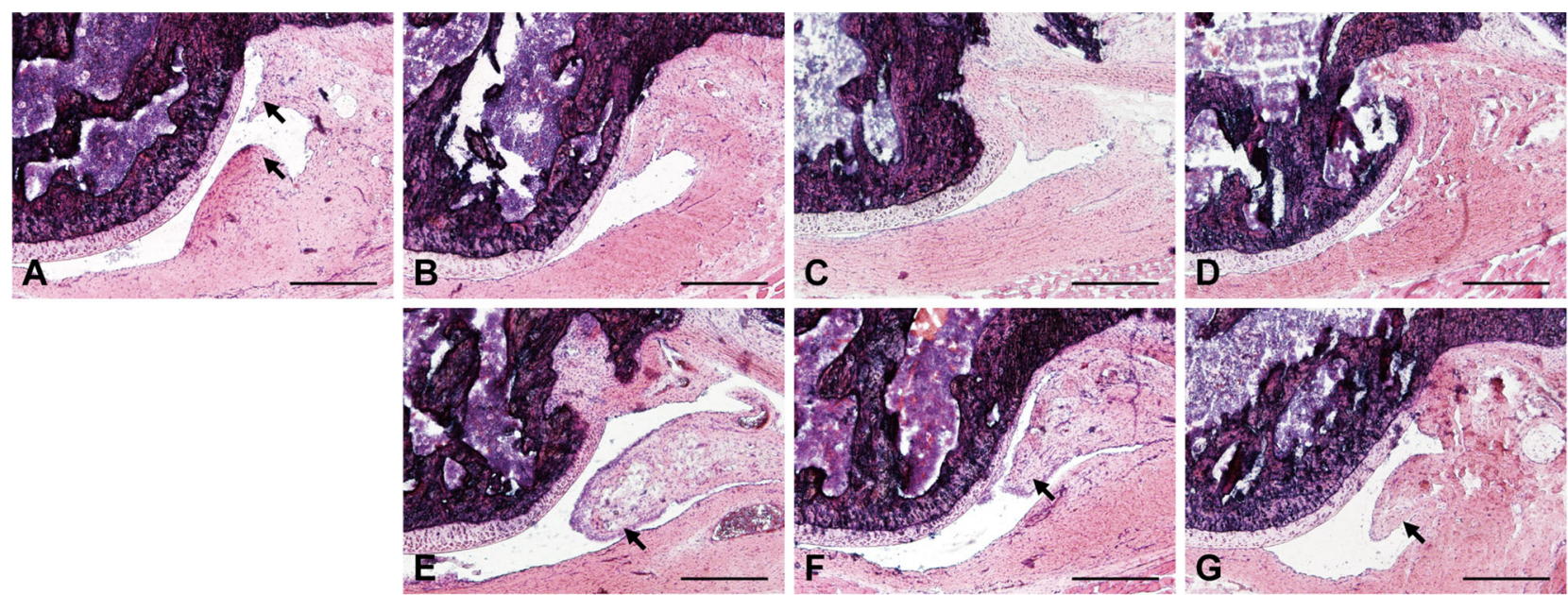
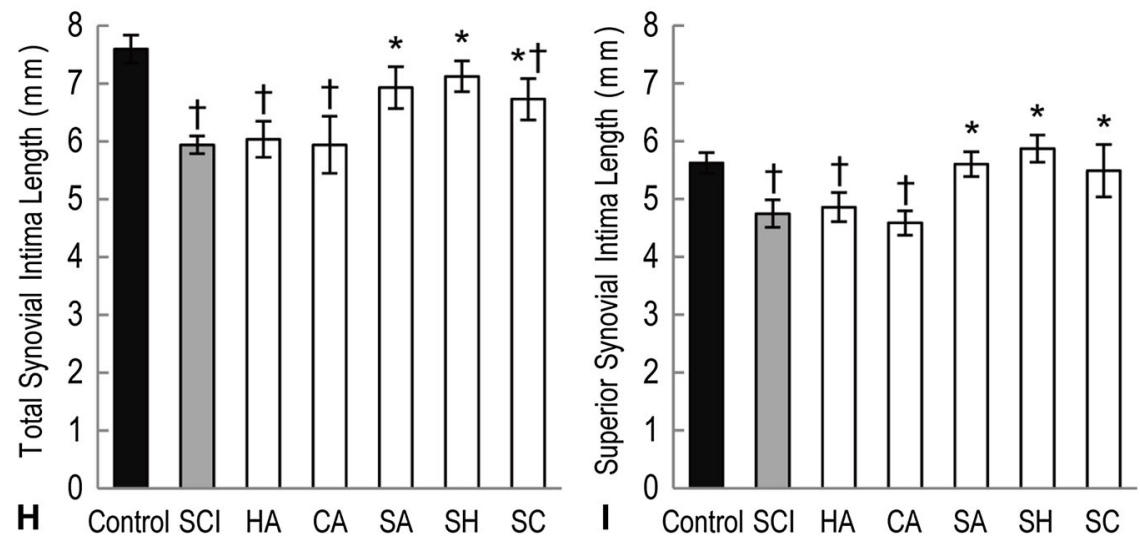

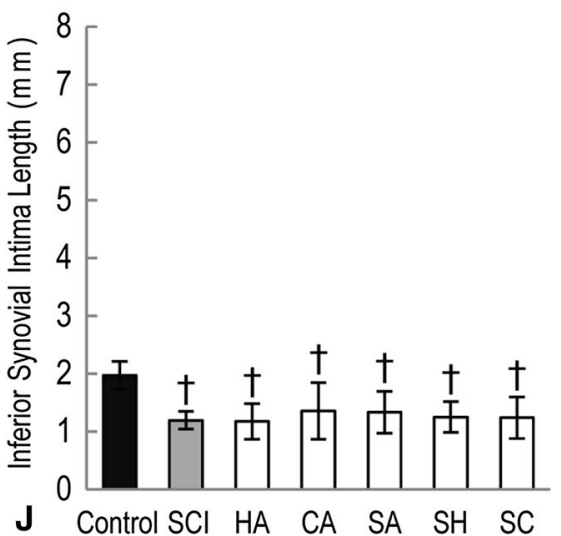

Fig. 3A-J The posterior synovial intima length was measured on the histologic sections stained with hematoxylin and eosin in the (A) control group, and the (B) spinal cord injury, (C) heat alone, (D) cold alone, (E) stretching alone, (F) stretching after heat, and (G) stretching after cold groups. The synovial folds are observed in the stretching alone, stretching after heat, and stretching after cold groups (arrows). Bars $=400 \mu \mathrm{m}$. The $(\mathbf{H})$ total and (I) posterosuperior

and providing equivalency of sample size for statistical purposes. Nonetheless, this reduces sample size (the number of rats) and cannot preclude chance findings attributable to intraindividual and interindividual variation. Finally, the results obtained in this study are limited to rats with spinal cord injury who were under anesthesia. Although anesthesia alters muscle tone and has been found to enable greater manipulation of contracted joints, this animal study dealt with the resultant alterations but not varying muscle tone depending on conditions. However, whether it can be generalized to awake humans with neurologic disorders is unclear but warrants further investigations.

Contractures are defined as limitations in the ROM of affected joints. The contractures developed 3 weeks after spinal cord injury in this experiment, and this is consistent with results of previous animal studies that ROM restrictions were observed from the first 2 weeks $[32,33]$. In the synovial intima lengths in the stretching alone, stretching after heat, and stretching after cold groups were longer than that in the spinal cord injury group, but not the $(\mathbf{J})$ posteroinferior length. $\mathrm{SCI}=$ spinal cord injury; $\mathrm{HA}=$ heat alone; $\mathrm{CA}=$ cold alone; $\mathrm{SA}=$ stretching alone; $\mathrm{SH}=$ stretching after heat; $\mathrm{SC}=$ stretching after cold. ${ }^{*}$ Greater than the spinal cord injury group and †Less than the control group.

current study, stretching, which is applied for 1 week from the third postoperative week, restored the limitation in ROM, suggesting that stretching is effective in treating contractures not only early after spinal cord injury in rats [29], but also after contractures developed. Although heat alone had no observable effect on ROM, stretching after heat led to a greater recovery of the loss of extension ROM in this animal model than stretching alone. In addition, stretching after heat achieved improvements in muscular and articular factors in contractures, whereas stretching alone did not. Usuba et al. [43] reported that stretching combined with infrared or ultrasound was more effective than stretching alone at increasing the ROM after immobilization in rats. Contracture development of rats with spinal cord injury has been reported to exhibit different progression patterns from those after immobilization [33], but its response to heat may be similar. It is known that heat reduces viscoelasticity of muscle tissue [34] and 

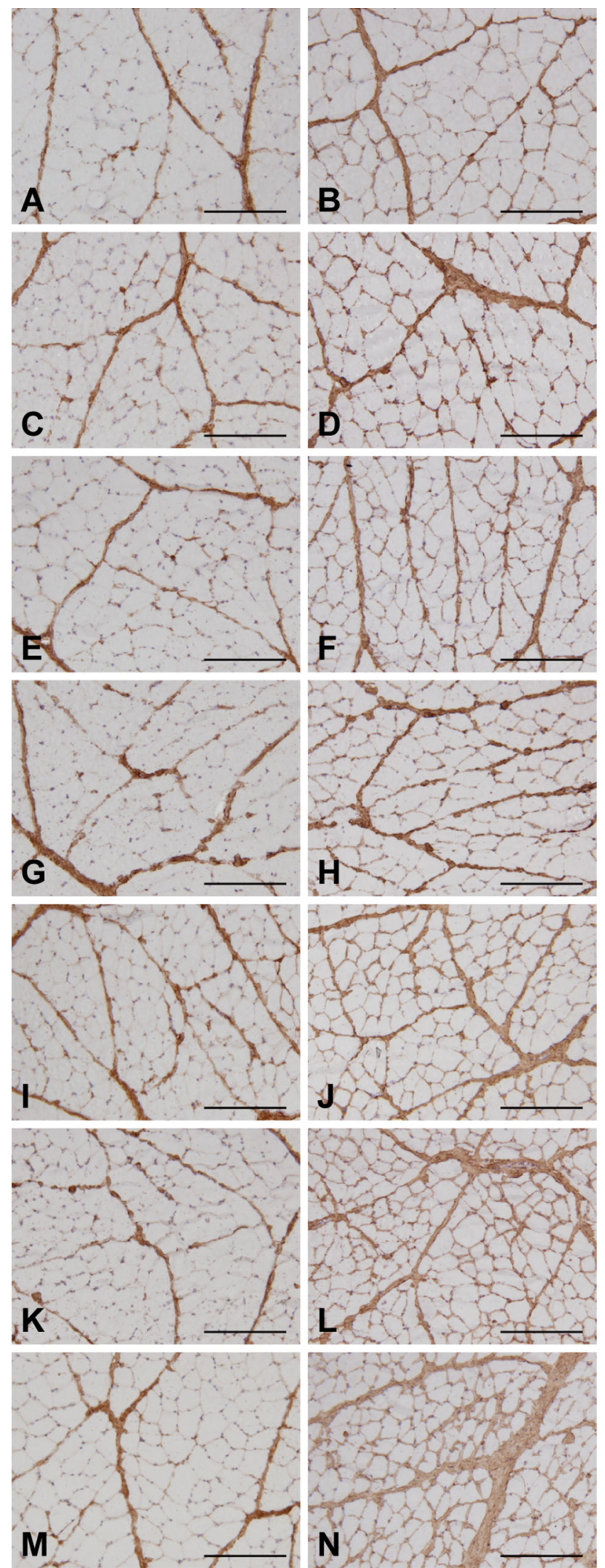

Fig. 4A-N The staining intensity and pattern showed no difference among the (A) control group for collagen Type I and (B) Type III; (C) the spinal cord injury group for collagen Type I and (D) Type III; (E) heat alone for collagen Type 1 and (F) Type III; (G) cold alone for collagen Type I and (H) Type III; (I) stretching alone for collagen Type I and (J) Type III; (K) stretching after heat for collagen Type I and (L) Type III; and (M) the stretching after cold collagen Type I and (N) Type III groups. Bars $=200 \mu \mathrm{m}$.

increases extensibility of collagenous tissue [23, 45]. Under warm conditions, the increase in extensibility of soft tissue around the joints would result in increased effectiveness of stretching. Unlike thermal therapy, there was no difference in ROM between stretching alone and stretching after cold in this animal study. Moreover, stretching after cold did not improve the muscular contracture. Therapeutic cold is considered to be more effective in reducing spasticity than heat [39] but it increases viscoelasticity of muscle tissue [34]. These conflicting effects may result in less effectiveness of cold than heat. The percentage improvement of the muscular factor was inferior to that of the articular factor in most of the treatment protocols in this animal study. Earlier studies showed that skeletal muscles in hind limbs of rats with spinal cord injury exhibit spasticity and are unable to contract voluntary [32,33]. A spinal cord injury may elicit considerable changes in muscle tissues through progression of contracture, thereby increasing the resistance to the treatments.

Some studies reported muscular fibrosis after central or peripheral nervous system disorders $[2,35]$ and are consistent with our results. In our animal study, muscular fibrosis after spinal cord injury was not improved by any treatments, but stretching after heat improved the muscular factor of contractures. Although fibrosis is one of the important histopathologic features of muscle tissues after spinal cord injury, we propose that correction of muscle shortening can be achieved without removal of fibrosis. Generally, collagen Type I in muscle tissue is associated with stiffness, and the abundance of collagen Type III correlates with tissue compliance [18]. Increased total collagen content and accumulation of collagen Type I in endomysium in spastic muscles of children with cerebral palsy have been reported [2]. Conversely, there were no differences in the intensity and distribution of immunostaining for collagen Types I and III among any groups in this animal study. This difference may be attributed to differences in species, age, model, and/or disease duration. Factors other than expression of collagen Types I and III may be responsible for muscular contractures in early-stage spinal cord injury. 
In the current study and a previous animal study [29], stretching improved the joint capsule shortening after spinal cord injury. Heat and cold alone had no effect on the synovial intima length. Improvements in the articular factor of contracture appeared to be associated with increases in synovial length. Therefore, shortening of the joint capsule seen in this study may be partly responsible for the articular factor in rats with spinal cord injury, supporting results of previous studies [29, 31].

Findings from the current study imply that heat or cold alone is ineffective and stretching is helpful for correction of contractures after spinal cord injury in rats. In addition, we provide evidence that heat is more beneficial than cold to increase the effectiveness of stretching for the treatment of contractures after spinal cord injury. The treatments evaluated in our study, using this particular animal model, should be considered to be geared toward treatment of contractures after an experimentally induced spinal cord injury. We believe it is reasonable to consider preliminary clinical studies to determine whether stretching after heat is clinically applicable to contractures derived from spastic paralyses.

Acknowledgments We thank Yuki Takahashi BSc, and Hitomi Atsuji BSc (School of Health Sciences, Faculty of Medicine, Hiroshima University), for their skilled technical assistance.

\section{References}

1. Ando A, Hagiwara Y, Onoda Y, Hatori K, Suda H, Chimoto E, Itoi E. Distribution of type A and B synoviocytes in the adhesive and shortened synovial membrane during immobilization of the knee joint in rats. Tohoku J Exp Med. 2010;221:161-168.

2. Booth CM, Cortina-Borja MJ, Theologis TN. Collagen accumulation in muscles of children with cerebral palsy and correlation with severity of spasticity. Dev Med Child Neurol. 2001;43:314320.

3. Botte MJ, Nickel VL, Akeson WH. Spasticity and contracture: physiologic aspects of formation. Clin Orthop Relat Res. 1988;233:7-18.

4. Bovend'Eerdt TJ, Newman M, Barker K, Dawes H, Minelli C, Wade DT. The effects of stretching in spasticity: a systematic review. Arch Phys Med Rehabil. 2008;89:1395-1406.

5. Cooper JE, Shwedyk E, Quanbury AO, Miller J, Hildebrand D. Elbow joint restriction: effect on functional upper limb motion during performance of three feeding activities. Arch Phys Med Rehabil. 1993;74:805-809.

6. Coutinho EL, DeLuca C, Salvini TF, Vidal BC. Bouts of passive stretching after immobilization of the rat soleus muscle increase collagen macromolecular organization and muscle fiber area. Connect Tissue Res. 2006;47:278-286.

7. Dalyan M, Sherman A, Cardenas DD. Factors associated with contractures in acute spinal cord injury. Spinal Cord. 1998;36:405-408.

8. Diong J, Harvey LA, Kwah LK, Eyles J, Ling MJ, Ben M, Herbert RD. Incidence and predictors of contracture after spinal cord injury: a prospective cohort study. Spinal Cord. 2012;50: 579-584.
9. Fergusson D, Hutton B, Drodge A. The epidemiology of major joint contractures: a systematic review of the literature. Clin Orthop Relat Res. 2007;456:22-29.

10. Fox P, Richardson J, McInnes B, Tait D, Bedard M. Effectiveness of a bed positioning program for treating older adults with knee contractures who are institutionalized. Phys Ther. 2000;80:363372.

11. Gomes AR, Cornachione A, Salvini TF, Mattiello-Sverzut AC. Morphological effects of two protocols of passive stretch over the immobilized rat soleus muscle. J Anat. 2007;210:328-335.

12. Grover J, Gellman H, Waters RL. The effect of a flexion contracture of the elbow on the ability to transfer in patients who have quadriplegia at the sixth cervical level. J Bone Joint Surg Am. 1996;78:1397-1400.

13. Hadi AM, Mouchaers KT, Schalij I, Grunberg K, Meijer GA, Vonk-Noordegraaf A, van der Laarse WJ, Beliën JA. Rapid quantification of myocardial fibrosis: a new macro-based automated analysis. Cell Oncol (Dordr). 2011;34:343-354.

14. Harvey LA, Crosbie J. Weight bearing through flexed upper limbs in quadriplegics with paralyzed triceps brachii muscles. Spinal Cord. 1999;37:780-785.

15. Harvey L, Crosbie J. Effect of elbow flexion contractures on the ability of people with C5 and C6 tetraplegia to lift. Physiother Res Int. 2001;6:76-82.

16. Harvey LA, Herbert RD. Muscle stretching for treatment and prevention of contracture in people with spinal cord injury. Spinal Cord. 2002;40:1-9.

17. Hedges LV, Ingram O. Statistical Methods for Meta-analysis. Orlando, FL: Harcourt Brace; 1985.

18. Hibino I, Okita M, Inoue T, Banno Y, Hoso M. Effect of immobilization on insoluble collagen concentration and type I and type III collagen isoforms of rat soleus muscle. J Jpn Phys Ther Assoc. 2008;11:1-6.

19. Kagaya H, Sharma M, Kobetic R, Marsolais EB. Ankle, knee, and hip moments during standing with and without joint contractures: simulation study for functional electrical stimulation. Am J Phys Med Rehabil. 1998;77:49-54.

20. Katalinic OM, Harvey LA, Herbert RD. Effectiveness of stretch for the treatment and prevention of contractures in people with neurological conditions: a systematic review. Phys Ther. 2011;91:11-24.

21. Kawamoto T. Use of a new adhesive film for the preparation of multi-purpose fresh-frozen sections from hard tissues, wholeanimals, insects and plants. Arch Histol Cytol. 2003;66:123-143.

22. Lee SH, Kayser V, Desmeules J, Guilbaud G. Differential action of morphine and various opioid agonists on thermal allodynia and hyperalgesia in mononeuropathic rats. Pain. 1994;57:233-240.

23. Lehmann JF, Masock AJ, Warren CG, Koblanski JN. Effect of therapeutic temperatures on tendon extensibility. Arch Phys Med Rehabil. 1970;51:481-487.

24. Lehmann JF, Warren CG, Scham SM. Therapeutic heat and cold. Clin Orthop Relat Res. 1974;99:207-245.

25. Lieber RL, Steinman S, Barash IA, Chambers H. Structural and functional changes in spastic skeletal muscle. Muscle Nerve. 2004;29:615-627.

26. Mollinger LA, Steffen TM. Knee flexion contractures in institutionalized elderly: prevalence, severity, stability, and related variables. Phys Ther. 1993;73:437-444.

27. Moriyama H, Kanemura N, Brouns I, Pintelon I, Adriaensen D, Timmermans JP, Ozawa J, Kito N, Gomi T, Deie M. Effects of aging and exercise training on the histological and mechanical properties of articular structures in knee joints of male rat. Biogerontology. 2012;13:369-381.

28. Moriyama H, Nishihara K, Hosoda M, Saka Y, Kanemura N, Takayanagi K, Yoshimura O, Tobimatsu Y. Contrasting alteration patterns of different cartilage plates in knee articular 
cartilage after spinal cord injury in rats. Spinal Cord. 2009;47:218-224.

29. Moriyama H, Tobimatsu Y, Ozawa J, Kito N, Tanaka R. Amount of torque and duration of stretching affects correction of knee contracture in a rat model of spinal cord injury. Clin Orthop Relat Res. 2013;471:3626-3636.

30. Moriyama H, Yoshimura O, Kawamata S, Takayanagi K, Kurose T, Kubota A, Hosoda M, Tobimatsu Y. Alteration in articular cartilage of rat knee joints after spinal cord injury. Osteoarthritis Cartilage. 2008;16:392-398.

31. Moriyama H, Yoshimura O, Kawamata S, Takemoto H, Saka Y, Tobimatsu Y. Alteration of knee joint connective tissues during contracture formation in spastic rats after an experimentally induced spinal cord injury. Connect Tissue Res. 2007;48:180187.

32. Moriyama H, Yoshimura O, Sunahori H, Nitta H, Imakita H, Saka Y, Maejima H, Tobimatsu Y. Progression and direction of contractures of knee joints following spinal cord injury in the rat. Tohoku J Exp Med. 2004;204:37-44.

33. Moriyama H, Yoshimura O, Sunahori H, Tobimatsu Y. Comparison of muscular and articular factors in the progression of contractures after spinal cord injury in rats. Spinal Cord. 2006;44:174-181.

34. Mutungi G, Ranatunga KW. Temperature-dependent changes in the viscoelasticity of intact resting mammalian (rat) fast- and slow-twitch muscle fibres. J Physiol. 1998;508:253-265.

35. Nikolaou S, Liangjun H, Tuttle LJ, Weekley H, Christopher W, Lieber RL, Cornwall R. Contribution of denervated muscle to contractures after neonatal brachial plexus injury: not just muscle fibrosis. Muscle Nerve. 2014;49:398-404.

36. Renner AF, Carvalho E, Soares E, Mattiello-Rosa S. The effect of a passive muscle stretching protocol on the articular cartilage. Osteoarthritis Cartilage. 2006;14:196-202.
37. Scott JA, Donovan WH. The prevention of shoulder pain and contracture in the acute tetraplegia patient. Paraplegia. 1981;19:313-319.

38. Tabary JC, Tabary C, Tardieu C, Tardieu G, Goldspink G. Physiological and structural changes in the cat's soleus muscle due to immobilization at different lengths by plaster casts. $J$ Physiol. 1972;224:231-244.

39. Tepperman SP, Devlin M. The therapeutic use of local heat and cold. Can Fam Physician. 1986;32:1110-1114.

40. Trudel G, Seki M, Uhthoff HK. Synovial adhesions are more important than pannus proliferation in the pathogenesis of knee joint contracture after immobilization: an experimental investigation in the rat. J Rheumatol. 2000;27:351-357.

41. Trudel G, Uhthoff HK. Contractures secondary to immobility: is the restriction articular or muscular? An experimental longitudinal study in the rat knee. Arch Phys Med Rehabil. 2000;81:6-13.

42. Usuba M, Akai M, Shirasaki Y, Miyakawa S. Experimental joint contracture correction with low torque: long duration repeated stretching. Clin Orthop Relat Res. 2007;456:70-78.

43. Usuba M, Miyanaga Y, Miyakawa S, Maeshima T, Shirasaki Y. Effect of heat in increasing the range of knee motion after the development of a joint contracture: an experiment with an animal model. Arch Phys Med Rehabil. 2006;87:247-253.

44. Vasilceac FA, Renner AF, Teodoro WR, Mattiello-Rosa SM. The remodeling of collagen fibers in rats ankles submitted to immobilization and muscle stretch protocol. Rheumatol Int. 2011;31:737-742.

45. Warren CG, Lehmann JF, Koblanski JN. Elongation of rat tail tendon: effect of load and temperature. Arch Phys Med Rehabil. 1971;52:465-474.

46. Yarkony GM, Bass LM, Keenan V 3rd, Meyer PR Jr. Contractures complicating spinal cord injury: incidence and comparison between spinal cord centre and general hospital acute care. Paraplegia. 1985;23:265-271. 